\title{
Control of the Nonlinear Wave-Type Dynamics Using the Derivative-Free Nonlinear Kalman Filter
}

\author{
G. Rigatos ${ }^{1} \cdot$ P. Siano ${ }^{2}$
}

Received: 24 December 2015 / Accepted: 28 February 2016 / Published online: 21 April 2016

(C) Springer Science+Business Media Singapore 2016

\begin{abstract}
The objective of the paper is to develop a pointwise control method for a $1 \mathrm{D}$ nonlinear wave equation and a filtering approach for estimating the dynamics of such a system from measurements provided from a small number of sensors. It is shown that the numerical solution of the associated partial differential equation results into a set of nonlinear ordinary differential equations. With the application of a diffeomorphism that is based on differential flatness theory it is shown that an equivalent description of the system in the linear canonical (Brunovsky) form is obtained. This transformation enables to obtain estimates about the state vector of the system through the application of the standard Kalman Filter recursion. For the local subsystems, into which the nonlinear wave equation is decomposed, it becomes possible to apply pointwise state estimation-based feedback control. The efficiency of the proposed filtering and control approach for nonlinear systems described by $1 \mathrm{D}$ partial differential equations of the wave type (e.g. sine-Gordon PDE) is confirmed through simulation experiments. It is shown that, by applying feedback control, the nonlinear wave-type dynamics can be made to track any reference setpoint.
\end{abstract}

Keywords Differential flatness theory · Derivative-free nonlinear Kalman Filter · Distributed parameter systems . Nonlinear feedback control $\cdot$ Nonlinear wave equations

\footnotetext{
G. Rigatos

grigat@ieee.org

P. Siano

psiano@unisa.it

1 Unit of Industrial Automation, Industrial Systems Institute, 26504 Rion Patras, Greece

2 Department of Industrial Engineering, University of Salerno, 84084 Fisciano, Italy
}

\section{Introduction}

The problem of state estimation and control of distributed parameter systems described by partial differential equations of the parabolic, hyperbolic and elliptic type is of advanced difficulty comparing to control of lumped parameter systems, the latter being modeled in the form of ordinary differential equations [1-3]. The problem becomes more difficult in case that the distributed parameter systems are characterized by nonlinearities [4-9]. The paper treats the problem of piecewise control of nonlinear wave-type partial differential equations. Control of nonlinear wave dynamics is met in several applications. For instance, nonlinear wavetype differential equations is met in communication systems (transmission lines, optical fibers and electromagnetic waves propagation), in electronics (Josephson junctions), in fluid flow models, in structural engineering (dynamic analysis of buildings under seismic waves, mechanical structures subjected to vibrations, pendulum chains), in biomedical systems (voltage propagation and variations in neuron's membrane), etc. Solving nonlinear estimation and control problems for such systems is important for modifying their dynamics and for succeeding their functioning according to specifications [10-17].

Following the procedure for numerical solution of the nonlinear PDE of the wave-type dynamics, a set of coupled nonlinear ordinary differential equations is obtained and written in a state-space form [18]. For the latter state-space description, differential flatness properties are proven. Thus, it is shown that all state variables and the control inputs of the state-space model can be written as functions of a vector of algebraic variables that constitute the flat output änd also as functions of the flat output's derivatives [19-23]. By applying a change of coordinates (diffeomorphism) which is based on differential flatness theory it is shown that the state- 
space model of the wave-type PDE can be written in the linear canonical (Brunovsky) form, in which the previously noted nonlinear ordinary differential equations are now transformed into linear ones. Next, pointwise feedback control is applied to the wave-type PDE. For each local linear model of the aforementioned differential equations the state feedback control is selected such that asymptotic stability is assured. This can be done using for instance pole-placement methods.

Another objective of the article is to implement statefeedback control of the nonlinear wave dynamics using measurements from a small number of sensors $[24,25]$. This implies that for state vector elements of the PDE's state-space description which cannot be measured directly, state estimation with filtering methods has to be be applied. Filtering for nonlinear distributed parameter systems is again a non-trivial problem [26-29]. Both observer-based and Kalman Filterbased approaches have been proposed [30-34]. To this end, in this paper, a new nonlinear filtering method, under the name Derivative-free nonlinear Kalman Filtering, is proposed. The filter consists of the standard Kalman Filter recursion applied to the linear equivalent state-space model of the wave PDE [35-37]. Moreover, an inverse transformation which is based on differential flatness properties enables to obtain estimates of the state variables of the initial system's description.

The structure of the paper is as follows: in "Nonlinear 1D wave-type partial differential equations" section, models of nonlinear 1D wave-type partial differential equations are examined, while emphasis is given to the sine-Gordon PDE model. In "Differential flatness theory and transformation into canonical forms" section, the use of differential flatness theory for transforming nonlinear dynamical systems into the linear and canonical state-space form is explained. In "State-space description of the nonlinear wave dynamics" section a state-space description is obtained for the model of the nonlinear wave-type dynamics. In "Solution of the control and estimation problem for nonlinear wave dynamics" section, the previous linearization transformation is applied to control and filtering of distributed parameter systems. In "Simulation tests" section simulation tests are carried out to evaluate the proposed control and filtering approach for distributed parameter systems. Finally, in "Conclusions" section concluding remarks are stated.

\section{Nonlinear 1D Wave-Type Partial Differential Equations}

\subsection{Sine-Gordon Nonlinear PDE in Coupled Nonlinear Pendula}

Nonlinear 1D wave-type partial differential equations appear in models of coupled oscillators. One can consider for example the forced damped sine-Gordon equation [10] $\frac{\partial^{2} \phi}{\partial t^{2}}+c \frac{\partial \phi}{\partial t}-k \frac{\partial^{2} \phi}{\partial x^{2}}+\sin (\phi)=l$

where $c$ and $l$ are constants. This type of PDE appears in many physical phenomena, such nonlinear resonant optics and Josephson junctions, or as a dynamic model of electrons in a crystal lattice. Equation (1) describes the motion of an array of pendula each of which is coupled to its nearest neighbors by a torsional spring with a coupling coefficient $k$. Each pendulum is subject to a constant torque $l$ and to a viscous drag force with coefficient $c$. The angle $x_{i}$ of the $i$-th pendulum and the vertical axis evolves according to Eq. (1).

By considering a one dimensional grid of $N$ sample points and by computing the second-order derivative

$\frac{\partial^{2} \phi}{\partial x^{2}} \simeq \frac{1}{h^{2}}\left(\phi_{i+1}-2 \phi_{i}+\phi_{i-1}\right)$

one has the decomposition of the nonlinear partial differential equation into a set of nonlinear ordinary differential equations of the form

$\ddot{x}_{i}+c \dot{x}_{i}+\epsilon \sin \left(x_{i}\right)=k\left(x_{i+1}-2 x_{i}+x_{i-1}+l\right) \quad i=1,2, \ldots$

One can also use a periodicity condition

$x_{i+N}=x_{i}+2 \pi$

The periodicity condition reduces the system from infinite many pendula into one with $N$ degrees of freedom

$$
\begin{aligned}
& \ddot{x}_{1}+c x_{1}+\epsilon \sin \left(x_{1}\right)=x_{2}+x_{N}-2 x_{1}+l-2 \pi \\
& \ddot{x}_{2}+c x_{2}+\epsilon \sin \left(x_{2}\right)=x_{3}+x_{1}-2 x_{2}+l \\
& \ldots \\
& \ddot{x}_{N}+c x_{N}+\epsilon \sin \left(x_{N}\right)=x_{1}+x_{N}-2 x_{N}+l+2 \pi
\end{aligned}
$$

\subsection{Sine-Gordon Nonlinear PDE in the Model of the Josephson Junction}

A transmission line is considered where transverse electromagnetic waves propagate as shown in Fig. 1. The transmission line consists of inductors, capacitors and Josephson junctions, such that for a length $d x$ of the transmission line the capacitance is $d C=C d x$, the inductance is $d L=L d x$ and the critical current is $d I_{0}=I_{0} d x$ [10]. Dividing the differential voltage drop $d V$ and the shunt current $d I$ by $d x$, one obtains

$$
\begin{aligned}
& \frac{\partial V}{\partial x}=-L \frac{\partial I}{\partial t} \\
& \frac{\partial I}{\partial x}=-C \frac{\partial V}{\partial t}-I_{0} \sin (\phi)
\end{aligned}
$$




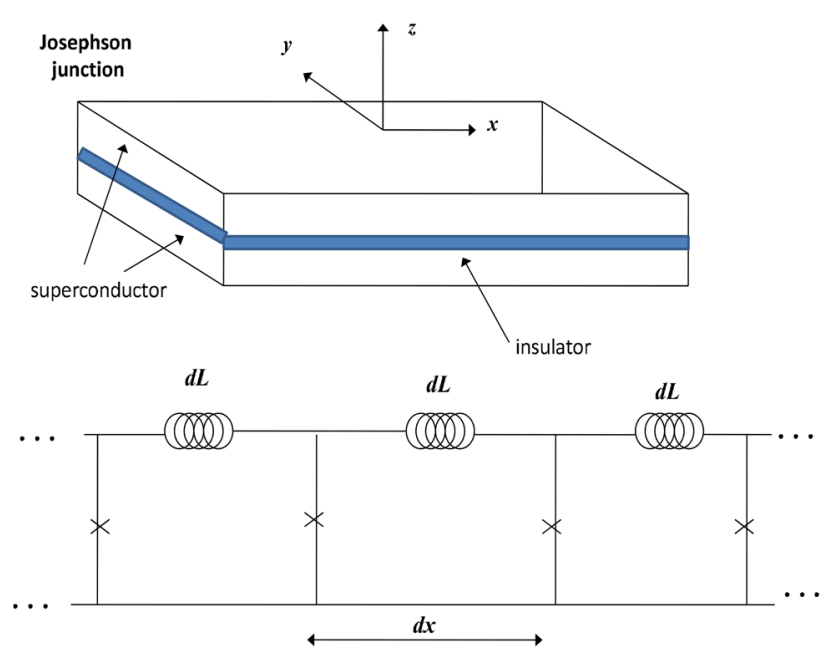

Fig. 1 Josephson transmission line described by Josephson junctions (top), a capacitance per unit length $C$, an inductance per unit length $L$ and a critical current per unit length $I_{0}$

where $\phi$ is the difference $\phi=\phi_{\text {upper }}-\phi_{\text {lower }}$ of the superconducting phases. The voltage is related to the rate of change of $\phi$ through the Josepshon equation

$\frac{\partial \phi}{\partial t}=\frac{2 e V}{h}$

and equivalently

$\frac{\partial \phi}{\partial x}=-\frac{2 e V}{h} I$

Thus, one arrives at the equation

$\frac{1}{c^{2}} \frac{\partial^{2} \phi}{\partial t^{2}}-\frac{\partial^{2} \phi}{\partial x^{2}}+\frac{1}{\lambda_{j}^{2}} \sin (\phi)=0$

where $c=(L C)^{-1 / 2}$ is the Swihart velocity and $\lambda_{j}=$ $\left(\hbar / 2 e L I_{0}\right)^{1 / 2}$ is the Josephson length. By rescaling lengths $\lambda_{j}$ and $\lambda_{j} / c$ one arrives at the sine-Gordon equation

$\frac{\partial^{2} \phi}{\partial t^{2}}+c \frac{\partial \phi}{\partial t}-\frac{\partial^{2} \phi}{\partial x^{2}}+\sin (\phi)=l$

\subsection{Current Equation in a Josepshon Transmission Line}

A wire of length $l$ is considered which is interrupted by identical Josephson junctions at positions denoted as $x_{1}, x_{2}, \ldots, x_{N}$. The partial differential equation governing the dynamics of the Josephson transmission line can be determined by considering the finite element representation shown in Fig. 2. Each inductor-capacitor segment represents a short

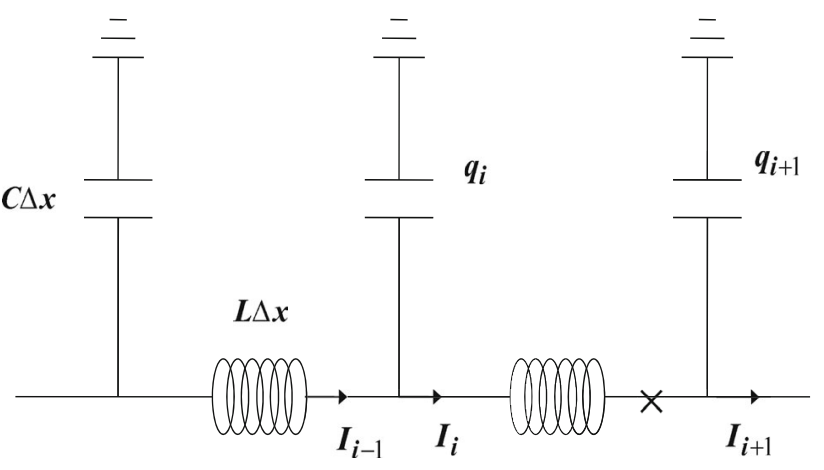

Fig. 2 Finite element representation of the Josephson transmission line ( $\times$ denotes a Josephson junction)

length $\Delta x$ of the wire. Some segments also contain a Josephson junction in series with the inductor.

At the $i$-th segment it holds that

$I_{i-1}=\dot{q}_{i}+I_{i}$

where $q_{i}$ is the charge on the $i$-th capacitor and $I_{i}$ is the current in the $i$-th inductor. Equating voltage drops of the two paths from node $i$ to ground it holds

$\frac{q_{i}}{C \Delta x}=L \Delta x \dot{I}_{i}+\frac{q_{i+1}}{C \Delta x}+\frac{\hbar}{2 e} \dot{\phi}_{j} \delta_{i, i_{j}}$

where $\hbar$ is Planck's constant divided by $2 \pi, e$ is the magnitude of the electronic charge and $\phi_{j}$ is the difference in the phase of the macroscopic quantum wave function across the $j$-th junction. The Kronecker $\delta$ term denotes the voltage drop across the $j$-th Josephson junction if it appears in segment $i$ of the transmission line. From Eqs.(11) and (12) one obtains

$v^{2} \frac{I_{i+1}-2 I_{i}+I_{i-1}}{\Delta x^{2}}=\ddot{I}_{i}+\frac{\hbar}{2 e L \Delta x} \ddot{\phi}_{j} \delta_{i, i_{j}}$

$v^{2}=1 /(L C)$. Setting the limit $\Delta x \rightarrow 0$ yields an equation for the current $I(x, t)$

$\frac{\partial^{2} I}{\partial t^{2}}-v^{2} \frac{\partial^{2} I}{\partial x^{2}}+\sum_{i=1}^{N} \frac{\hbar}{2 e L} \ddot{\phi}_{j} \delta\left(x-x_{j}\right)=0$

The associated boundary conditions are $I(0, t)=I(l, t)$ $=I_{b}$. Moreover, about the dynamics of phase $\phi$ at the $j$-th Josephson junction one has

$\frac{\hbar C_{J}}{2 e} \ddot{\phi}_{j}+\frac{\hbar}{2 e R_{j}} \dot{\phi}_{j}+I_{c} \sin (\phi)_{j}=I\left(x_{j}, t\right)$

where $I\left(x_{j}, t\right)$ is the current through the $j$-th junction. The parameters of the junction are the capacitance $C_{j}$, the resistance $R_{j}$ and the current $I_{c}$. 


\section{Differential Flatness Theory and Transformation into Canonical Forms}

\subsection{Definition of Differentially Flat Systems}

Differential flatness theory will be used to obtain a linearized state-space description of the nonlinear wave-type PDE. Subsequently, it will be used for solving the associated state estimation and control problems. Differential flatness theory is currently a main direction in the analysis of nonlinear dynamical systems. To conclude if a dynamical system is differentially flat, the following should be examined: (i) the existence of the so-called flat output, i.e. a new variable which is expressed as a function of the system's state variables. It should hold that the flat output and its derivatives should not be coupled in the form of an ordinary differential equation, (ii) the components of the system (i.e. state variables and control input) should be expressed as functions of the flat output and its derivatives [20-23].

The main principles of differential flatness theory are as follows [20-23]: A finite dimensional system is considered. This can be written in the general form of an ordinary differential equation (ODE), i.e. $S_{i}\left(w, \dot{w}, \ddot{w}, \ldots, w^{(i)}\right), \quad i=$ $1,2, \ldots, q$. The term $w$ denotes the system variables (these variables are for instance the elements of the system's state vector and the control input) while $w^{(i)}, i=1,2, \ldots, q$ are the associated derivatives. Such a system is said to be differentially flat if there is a collection of $m$ functions $y=\left(y_{1}, \ldots, y_{m}\right)$ of the system variables and of their time-derivatives, i.e. $y_{i}=\phi\left(w, \dot{w}, \ddot{w}, \ldots, w^{\left(\alpha_{i}\right)}\right), \quad i=$ $1, \cdots, m$ satisfying the following two conditions [20-23]: (1) There does not exist any differential relation of the form $R\left(y, \dot{y}, \ldots, y^{(\beta)}\right)=0$ which implies that the derivatives of the flat output are not coupled in the sense of an ODE, or equivalently it can be said that the flat output is differentially independent, (2) All system variables (i.e. the elements of the system's state vector $w$ and the control input) can be expressed using only the flat output $y$ and its time derivatives $w_{i}=\psi_{i}\left(y, \dot{y}, \ldots, y^{\left(\gamma_{i}\right)}\right), \quad i=1, \ldots, s$.

\subsection{Conditions for Applying Differential Flatness Theory}

Next, a new filtering and control method for distributed parameter systems will be developed, in accordance to the differential flatness theory. It will be shown that the proposed filtering and control methods can be efficiently applied to the model of the nonlinear wave-type PDE.

It will be shown that following established discretization procedures the model of the nonlinear wave-type PDE can be written as a state-space model of the form $\dot{x}=f(x, u)$. Moreover, the previous state-space description can be transformed to the form of an affine in-the-input system by adding an integrator to each input $[21,23]$. In the latter case one obtains

$\dot{x}=f(x)+\sum_{i=1}^{m} g_{i}(x) u_{i}$

The following definitions are now used [24]:

(i) Lie derivative $L_{f} h(x)$ stands for the Lie derivative $L_{f} h(x)=(\nabla h) f$ and the repeated Lie derivatives are recursively defined as $L_{f}^{0} h=h$ for $i=0, L_{f}^{i} h=$ $L_{f} L_{f}^{i-1} h=\nabla L_{f}^{i-1} h f$ for $i=1,2, \ldots$.

(ii) Lie Bracket $a d_{f}^{i} g$ stands for a Lie Bracket which is defined recursively as $a d_{f}^{i} g=\left[f, a d_{f}^{i-1}\right] g$ with $a d_{f}^{0} g=$ $g$ and $a d_{f} g=[f, g]=\nabla g f-\nabla f g$.

If the system of Eq. (16) can be linearized by a diffeomor$\operatorname{phism} z=\phi(x)$ and a static state feedback $u=\alpha(x)+\beta(x) v$ into the following form

$$
\begin{aligned}
& \dot{z}_{i, j}=z_{i+1, j} \quad \text { for } 1 \leq j \leq m \text { and } 1 \leq i \leq v_{j}-1 \\
& \dot{z}_{v_{i, j}}=v_{j}
\end{aligned}
$$

with $\sum_{j=1}^{m} v_{j}=n$, then $y_{j}=z_{1, j}$ for $1 \leq j \leq m$ are the 0 -flat outputs which can be written as functions of only the elements of the state vector $x$. To define conditions for transforming the system of Eq. (16) into the canonical form described in Eq. (17) the following theorem holds [23].

Theorem For nonlinear systems described by Eq. (16) the following variables are defined: (i) $G_{0}=\operatorname{span}\left[g_{1}, \ldots, g_{m}\right]$, (ii) $G_{1}=\operatorname{span}\left[g_{1}, \ldots, g_{m}, a d_{f} g_{1}, \ldots, a d_{f} g_{m}\right], \ldots(k)$ $G_{k}=\operatorname{span}\left\{a d_{f}^{j} g_{i}\right.$ for $\left.0 \leq j \leq k, \quad 1 \leq i \leq m\right\}$. Then, the linearization problem for the system of Eq. (16) can be solved if and only if: (1) The dimension of $G_{i}, i=1, \ldots, k$ is constant for $x \in X \subseteq R^{n}$ and for $1 \leq i \leq n-1$, (2) The dimension of $G_{n-1}$ if of order n, (3). The distribution $G_{k}$ is involutive for each $1 \leq k \leq n-2$.

\subsection{Transformation of the Nonlinear Wave-Type PDE into a Canonical State-Space Form}

It is assumed now that after defining the flat outputs of the previous state-space description of the nonlinear wave-type PDE and after expressing the system state variables and control inputs as functions of the flat output and of the associated derivatives, the system can be transformed in the Brunovsky canonical form: 


$$
\begin{aligned}
& \dot{x}_{1}=x_{2} \\
& \ldots \\
& \dot{x}_{r_{1}-1}=x_{r_{1}} \\
& \dot{x}_{r_{1}}=f_{1}(x)+\sum_{j=1}^{p} g_{1_{j}}(x) u_{j}+d_{1} \\
& \dot{x}_{r_{1}+1}=x_{r_{1}+2} \\
& \cdots \\
& \dot{x}_{p-1}=x_{p} \\
& \dot{x}_{p}=f_{p}(x)+\sum_{j=1}^{p} g_{p_{j}}(x) u_{j}+d_{p} \\
& y_{1}=x_{1} \\
& \cdots \\
& y_{p}=x_{n-r_{p}+1}
\end{aligned}
$$

where $x=\left[x_{1}, \ldots, x_{n}\right]^{T}$ is the state vector of the transformed system (according to the differential flatness formulation), $u=\left[u_{1}, \ldots, u_{p}\right]^{T}$ is the set of control inputs, $y=\left[y_{1}, \ldots, y_{p}\right]^{T}$ is the output vector, $f_{i}$ are the drift functions and $g_{i, j}, i, j=1,2, \ldots, p$ are smooth functions corresponding to the control input gains, while $d_{j}$ is a variable associated to external disturbances. It holds that $r_{1}+r_{2}+\cdots+r_{p}=n$. Having written the initial nonlinear system into the canonical (Brunovsky) form it holds

$y_{i}^{\left(r_{i}\right)}=f_{i}(x)+\sum_{j=1}^{p} g_{i j}(x) u_{j}+d_{j}$

Next the following vectors and matrices can be defined: $f(x)=\left[f_{1}(x), \ldots, f_{n}(x)\right]^{T}, g(x)=\left[g_{1}(x), \ldots, g_{n}(x)\right]^{T}$, with $g_{i}(x)=\left[g_{1 i}(x), \ldots, g_{p i}(x)\right]^{T}, A=\operatorname{diag}\left[A_{1}, \ldots, A_{p}\right]$, $B=\operatorname{diag}\left[B_{1}, \ldots, B_{p}\right], C^{T}=\operatorname{diag}\left[C_{1}, \ldots, C_{p}\right], \quad d=$ $\left[d_{1}, \ldots, d_{p}\right]^{T}$, where matrix $A$ has the MIMO canonical form, i.e. with block-diagonal elements

$$
\begin{aligned}
& A_{i}=\left(\begin{array}{cccc}
0 & 1 & \cdots & 0 \\
0 & 0 & \cdots & 0 \\
\vdots & \vdots & \cdots & \vdots \\
0 & 0 & \cdots & 1 \\
0 & 0 & \cdots & 0
\end{array}\right)_{r_{i} \times r_{i}} \\
& B_{i}^{T}=\left(\begin{array}{lllll}
0 & 0 & \cdots & 0 & 1
\end{array}\right)_{1 \times r_{i}} \\
& C_{i}=\left(\begin{array}{lllll}
1 & 0 & \cdots & 0 & 0
\end{array}\right)_{1 \times r_{i}}
\end{aligned}
$$

Thus, Eq. (19) can be written in state-space form

$$
\begin{aligned}
& \dot{x}=A x+B v+B \tilde{d} \\
& y=C x
\end{aligned}
$$

where the control input is written as $v=f(x)+g(x) u$. The system of Eqs. (20) and (21) is in controller and observer canonical form.

\subsection{Derivative-Free Nonlinear Kalman Filtering}

As mentioned above, for the system of Eq. (21), state estimation is possible by applying the standard Kalman Filter. The system is first turned into discrete-time form using common discretization methods and then the recursion of the linear Kalman Filter described in Eqs. (40) and (41) is applied.

If the derivative-free Kalman Filter is used in place of the Extended Kalman Filter then in the EKF equations the following matrix substitutions should be performed: $J_{\phi}(k) \rightarrow A_{d}, J_{\gamma}(k) \rightarrow C_{d}$, where matrices $A_{d}$ and $C_{d}$ are the discrete-time equivalents of matrices $A$ and $C$ which have been defined Eq. (21) and which appear also in the measurement and time update of the standard Kalman Filter recursion. Matrices $A_{d}$ and $C_{d}$ can be computed using established discretization methods. Moreover, the covariance matrices $P(k)$ and $P^{-}(k)$ are the ones obtained from the linear Kalman Filter update equations.

\section{State-Space Description of the Nonlinear Wave Dynamics}

The following nonlinear wave equation is considered

$$
\frac{\partial^{2} \phi}{\partial t^{2}}=k \frac{\partial^{2} \phi}{\partial x^{2}}+f(\phi)+u(x, t)
$$

where $u(x, t)$ is assumed to be the external control input. Using the approximation for the partial derivative

$\frac{\partial^{2} \phi}{\partial x^{2}} \simeq=\frac{\phi_{i+1}-2 \phi_{i}+\phi_{i-1}}{\Delta x^{2}}$

and considering spatial measurements of variable $\phi$ along axis $x$ at points $x_{0}+i \Delta x, \quad i=1,2, \ldots, N$ one has

$$
\frac{\partial^{2} \phi_{i}}{\partial t^{2}}=\frac{K}{\Delta x^{2}} \phi_{i+1}-\frac{2 K}{\Delta x^{2}} \phi_{i}+\frac{K}{\Delta x^{2}} \phi_{i-1}+f\left(\phi_{i}\right)+u\left(x_{i}, t\right)
$$

By considering the associated samples of $\phi$ given by $\phi_{0}, \phi_{1}, \ldots, \phi_{N}, \phi_{N+1}$ one has

$$
\begin{aligned}
\frac{\partial^{2} \phi_{1}}{\partial t^{2}} & =\frac{K}{\Delta x^{2}} \phi_{2}-\frac{2 K}{\Delta x^{2}} \phi_{1}+\frac{K}{\Delta x^{2}} \phi_{0}+f\left(\phi_{1}\right)+u\left(x_{1}, t\right) \\
\frac{\partial^{2} \phi_{2}}{\partial t^{2}} & =\frac{K}{\Delta x^{2}} \phi_{3}-\frac{2 K}{\Delta x^{2}} \phi_{2}+\frac{K}{\Delta x^{2}} \phi_{1}+f\left(\phi_{2}\right)+u\left(x_{2}, t\right) \\
\frac{\partial^{2} \phi_{3}}{\partial t^{2}} & =\frac{K}{\Delta x^{2}} \phi_{4}-\frac{2 K}{\Delta x^{2}} \phi_{3}+\frac{K}{\Delta x^{2}} \phi_{2}+f\left(\phi_{3}\right)+u\left(x_{3}, t\right)
\end{aligned}
$$


...

$$
\begin{aligned}
\frac{\partial^{2} \phi_{N-1}}{\partial t^{2}}= & \frac{K}{\Delta x^{2}} \phi_{N}-\frac{2 K}{\Delta x^{2}} \phi_{N-1}+\frac{K}{\Delta x^{2}} \phi_{N-2}+f\left(\phi_{N-1}\right) \\
& +u\left(x_{N_{1}}, t\right) \\
\frac{\partial^{2} \phi_{N}}{\partial t^{2}}= & \frac{K}{\Delta x^{2}} \phi_{N+1}-\frac{2 K}{\Delta x^{2}} \phi_{N}+\frac{K}{\Delta x^{2}} \phi_{N-1} \\
& +f\left(\phi_{N}\right)+u\left(x_{N}, t\right)
\end{aligned}
$$

By defining the following state vector

$$
x^{T}=\left(\phi_{1}, \phi_{2}, \ldots, \phi_{N}\right)
$$

one obtains the following state-space description

$$
\begin{gathered}
\ddot{x}_{1}=\frac{K}{\Delta x^{2}} x_{2}-\frac{2 K}{\Delta x^{2}} x_{1}+\frac{K}{\Delta x^{2}} \phi_{0}+f\left(x_{1}\right)+u\left(x_{1}\right) \\
\ddot{x}_{2}=\frac{K}{\Delta x^{2}} x_{3}-\frac{2 K}{\Delta x^{2}} x_{2}+\frac{K}{\Delta x^{2}} x_{1}+f\left(x_{2}\right)+u\left(x_{2}\right) \\
\ddot{x}_{3}=\frac{K}{\Delta x^{2}} x_{4}-\frac{2 K}{\Delta x^{2}} x_{3}+\frac{K}{\Delta x^{2}} x_{2}+f\left(x_{3}\right)+u\left(x_{3}\right) \\
\ldots \\
\ddot{x}_{N-1}=\frac{K}{\Delta x^{2}} x_{N}-\frac{2 K}{\Delta x^{2}} x_{N-1}+\frac{K}{\Delta x^{2}} x_{N-2} \\
\quad+f\left(x_{N-1}\right)+u\left(x_{N-1}\right) \\
\ddot{x}_{N}=\frac{K}{\Delta x^{2}} \phi_{N+1}-\frac{2 K}{\Delta x^{2}} x_{N}+\frac{K}{\Delta x^{2}} x_{N-1} \\
+f\left(x_{N}\right)+u\left(x_{N}\right)
\end{gathered}
$$

Next, the following state variables are defined

$$
\begin{aligned}
& y_{1, i}=x_{i} \\
& y_{2, i}=\dot{x}_{i}
\end{aligned}
$$

and the state-space description of the system becomes as follows

$$
\begin{aligned}
& \dot{y}_{1,1}=y_{2,1} \\
& \dot{y}_{2,1}=\frac{K}{\Delta x^{2}} y_{1,2}-\frac{2 K}{\Delta x^{2}} y_{1,1}+\frac{K}{\Delta x^{2}} \phi_{0}+f\left(y_{1,1}\right)+u\left(y_{1,1}\right) \\
& \dot{y}_{1,2}=y_{2,2} \\
& \dot{y}_{2,2}=\frac{K}{\Delta x^{2}} y_{1,3}-\frac{2 K}{\Delta x^{2}} y_{1,2}+\frac{K}{\Delta x^{2}} y_{1,1}+f\left(y_{1,2}\right)+u\left(y_{1,2}\right) \\
& \dot{y}_{1,3}=y_{2,3} \\
& \dot{y}_{2,3}=\frac{K}{\Delta x^{2}} y_{1,4}-\frac{2 K}{\Delta x^{2}} y_{1,3}+\frac{K}{\Delta x^{2}} y_{1,2}+f\left(y_{1,3}\right)+u\left(y_{1,3}\right) \\
& \text {... } \\
& \text {... } \\
& \dot{y}_{1, N-1}=y_{2, N-1} \\
& \dot{y}_{2, N-1}=\frac{K}{\Delta x^{2}} y_{1, N}-\frac{2 K}{\Delta x^{2}} y_{1, N-1}+\frac{K}{\Delta x^{2}} y_{1, N-2}+f\left(y_{1, N-1}\right) \\
& +u\left(y_{1, N-1}\right)
\end{aligned}
$$

$\dot{y}_{1, N}=y_{2, N}$

$$
\begin{aligned}
\dot{y}_{2, N}= & \frac{K}{\Delta x^{2}} \phi_{N+1}-\frac{2 K}{\Delta x^{2}} y_{1, N}+\frac{K}{\Delta x} y_{1, N-1} \\
& +f\left(y_{1, N}\right)+u\left(y_{1, N}\right)
\end{aligned}
$$

The dynamical system described in Eq. (29) is a differentially flat one with flat output defined as the vector $\tilde{y}=$ $\left[y_{1,1}, y_{1,2}, \ldots, y_{1, N}\right]$. Indeed all state variables can be written as functions of the flat output and its derivatives.

Moreover, by defining the new control inputs

$$
\begin{aligned}
& v_{1}=\frac{K}{\Delta x^{2}} y_{1,2}-\frac{2 K}{\Delta x^{2}} y_{1,1}+\frac{K}{\Delta x^{2}} \phi_{0}+f\left(y_{1,1}\right)+u\left(y_{1,1}\right) \\
& v_{2}=\frac{K}{\Delta x^{2}} y_{1,3}-\frac{2 K}{\Delta x^{2}} y_{1,2}+\frac{K}{\Delta x^{2}} y_{1,1}+f\left(y_{1,2}\right)+u_{y_{1,2}} \\
& v_{3}=\frac{K}{\Delta x^{2}} y_{1,4}-\frac{2 K}{\Delta x^{2}} y_{1,3}+\frac{K}{\Delta x^{2}} y_{1,2}+f\left(y_{1,3}\right)+u\left(y_{1,3}\right) \\
& \ldots \\
& v_{N-1}=\frac{K}{\Delta x^{2}} y_{1, N}-\frac{2 K}{\Delta x^{2}} y_{1, N-1}+\frac{K}{\Delta x^{2}} y_{1, N-2} \\
& \quad+f\left(y_{1, N-1}\right)+u\left(y_{1, N-1}\right) \\
& v_{N}=\frac{K}{\Delta x^{2}} \phi_{N+1}-\frac{2 K}{\Delta x^{2}} y_{1, N}+\frac{K}{\Delta x^{2}} y_{1, N-1} \\
& \quad+f\left(y_{1, N}\right)+u\left(y_{1, N}\right)
\end{aligned}
$$

the following state-space description is obtained

$$
\begin{aligned}
& \left(\begin{array}{c}
\dot{y}_{1,1} \\
\dot{y}_{2,1} \\
\dot{y}_{1,2} \\
\dot{y}_{2,2} \\
\cdots \\
\dot{y}_{1, N-1} \\
\dot{y}_{2, N-1} \\
\dot{y}_{1, N} \\
\dot{y}_{2, N}
\end{array}\right)=\left(\begin{array}{ccccccccc}
0 & 1 & 0 & 0 & \cdots & 0 & 0 & 0 & 0 \\
0 & 0 & 0 & 0 & \cdots & 0 & 0 & 0 & 0 \\
0 & 0 & 0 & 1 & \cdots & 0 & 0 & 0 & 0 \\
0 & 0 & 0 & 0 & \cdots & 0 & 0 & 0 & 0 \\
0 & 0 & 0 & 0 & \cdots & 0 & 0 & 0 & 0 \\
0 & 0 & 0 & 0 & \cdots & 0 & 0 & 0 & 0 \\
\cdots & \cdots & \cdots & \cdots & \cdots & \cdots & \cdots \\
0 & 0 & 0 & 0 & \cdots & 0 & 1 & 0 & 0 \\
0 & 0 & 0 & 0 & \cdots & 0 & 0 & 0 & 0 \\
0 & 0 & 0 & 0 & \cdots & 0 & 0 & 0 & 1 \\
0 & 0 & 0 & 0 & \cdots & 0 & 0 & 0 & 0
\end{array}\right)\left(\begin{array}{c}
y_{1,1} \\
y_{2,1} \\
y_{1,2} \\
y_{2,2} \\
\cdots \\
y_{1, N-1} \\
y_{2, N-1} \\
y_{1, N} \\
y_{2, N}
\end{array}\right) \\
& +\left(\begin{array}{cccccc}
0 & 0 & 0 & \cdots & 0 & 0 \\
1 & 0 & 0 & \cdots & 0 & 0 \\
0 & 0 & 0 & \cdots & 0 & 0 \\
0 & 1 & 0 & \cdots & 0 & 0 \\
0 & 0 & 0 & \cdots & 0 & 0 \\
0 & 0 & 1 & \cdots & 0 & 0 \\
\cdots & \cdots & \cdots & \cdots & \cdots \\
0 & 0 & 0 & \cdots & 0 & 0 \\
0 & 0 & 0 & \cdots & 1 & 0 \\
0 & 0 & 0 & \cdots & 0 & 0 \\
0 & 0 & 0 & \cdots & 0 & 1
\end{array}\right)\left(\begin{array}{c}
v_{1} \\
v_{2} \\
v_{3} \\
\cdots \\
v_{N-1} \\
v_{N}
\end{array}\right)
\end{aligned}
$$

By selecting measurements from a subset of points $x_{j} j \in[1,2$, $\ldots, m]$, the associated observation (measurement) equation becomes 


$$
\left(\begin{array}{l}
z_{1} \\
z_{2} \\
\cdots \\
z_{m}
\end{array}\right)=\left(\begin{array}{cccccc}
1 & 0 & 0 & \cdots & 0 & 0 \\
0 & 0 & 0 & \cdots & 0 & 0 \\
\cdots & \cdots & \cdots & \cdots \\
0 & 0 & 0 & \cdots & 1 & 0 \\
0 & 0 & 0 & \cdots & 0 & 0
\end{array}\right)\left(\begin{array}{c}
y_{1,1} \\
y_{2,1} \\
y_{1,2} \\
y_{2,2} \\
\cdots \\
y_{1, N} \\
y_{2, N}
\end{array}\right)
$$

Thus, in matrix form one has the following state-space description of the system

$\dot{\tilde{y}}=A \tilde{y}+B v$

$\tilde{z}=C \tilde{y}$

Moreover, denoting $a=\frac{K}{\Delta x^{2}}$ and $b=-\frac{2 K}{\Delta x^{2}}$, the initial description of the system given in Eq. (31) is rewritten as follows

$$
\begin{aligned}
& \left(\begin{array}{c}
\dot{y}_{1,1} \\
\dot{y}_{2,1} \\
\dot{y}_{1,2} \\
\dot{y}_{2,2} \\
\cdots \\
\dot{y}_{1, N-1} \\
\dot{y}_{2, N-1} \\
\dot{y}_{1, N} \\
\dot{y}_{2, N}
\end{array}\right)=\left(\begin{array}{cccccccccccccc}
0 & 1 & 0 & 0 & 0 & 0 & 0 & \cdots & 0 & 0 & 0 & 0 & 0 & 0 \\
b & 0 & a & 0 & 0 & 0 & 0 & \cdots & 0 & 0 & 0 & 0 & 0 & 0 \\
0 & 0 & 0 & 1 & 0 & 0 & 0 & \cdots & 0 & 0 & 0 & 0 & 0 & 0 \\
a & 0 & b & 0 & a & 0 & 0 & \cdots & 0 & 0 & 0 & 0 & 0 & 0 \\
0 & 0 & 0 & 0 & 0 & 1 & 0 & \cdots & 0 & 0 & 0 & 0 & 0 & 0 \\
0 & 0 & a & 0 & b & 0 & a & \cdots & 0 & 0 & 0 & 0 & 0 & 0 \\
\cdots & \cdots & \cdots & \cdots & \cdots & \cdots & \cdots & \cdots & \cdots & \cdots \\
0 & 0 & 0 & 0 & 0 & 0 & 0 & \cdots & 0 & 0 & 0 & 1 & 0 & 0 \\
0 & 0 & 0 & 0 & 0 & 0 & 0 & \cdots & 0 & 0 & b & 0 & a & 0 \\
0 & 0 & 0 & 0 & 0 & 0 & 0 & \cdots & 0 & 0 & 0 & 0 & 0 & 1 \\
0 & 0 & 0 & 0 & 0 & 0 & 0 & \cdots & 0 & 0 & a & 0 & b & 0
\end{array}\right)\left(\begin{array}{c}
y_{1,1} \\
y_{2,1} \\
y_{1,2} \\
y_{2,2} \\
\cdots \\
y_{1, N-1} \\
y_{2, N-1} \\
y_{1, N} \\
y_{2, N}
\end{array}\right) \\
& +\left(\begin{array}{cccccc}
0 & 0 & 0 & \cdots & 0 & 0 \\
1 & 0 & 0 & \cdots & 0 & 0 \\
0 & 0 & 0 & \cdots & 0 & 0 \\
0 & 1 & 0 & \cdots & 0 & 0 \\
0 & 0 & 0 & \cdots & 0 & 0 \\
0 & 0 & 1 & \cdots & 0 & 0 \\
\cdots & \cdots & \cdots & \cdots \\
0 & 0 & 0 & \cdots & 0 & 0 \\
0 & 0 & 0 & \cdots & 1 & 0 \\
0 & 0 & 0 & \cdots & 0 & 0 \\
0 & 0 & 0 & \cdots & 0 & 1
\end{array}\right)\left(\begin{array}{c}
v_{1} \\
v_{2} \\
v_{3} \\
\cdots \\
v_{N-1} \\
v_{N}
\end{array}\right)
\end{aligned}
$$

The associated control inputs are defined as

$$
\begin{aligned}
& v_{1}=\frac{K}{\Delta x^{2}} \phi_{0}+f\left(y_{1,1}\right)+u\left(y_{1,1}\right) \\
& v_{2}=f\left(y_{1,2}\right)+u\left(y_{1,2}\right) \\
& v_{3}=f\left(y_{1,3}\right)+u\left(y_{1,3}\right) \\
& \cdots \\
& v_{N-1}=f\left(y_{1, N-1}\right)+u\left(y_{1, N-1}\right) \\
& v_{N}=\frac{K}{\Delta x^{2}} \phi_{N+1}+f\left(y_{1, N}\right)+u\left(y_{1, N}\right)
\end{aligned}
$$

\section{Solution of the Control and Estimation Problem for Nonlinear Wave Dynamics}

\subsection{Solution of the Control Problem}

It holds that the dynamics of the linearized equivalent model of the nonlinear wave-type PDE takes the form

$$
\begin{aligned}
& \ddot{y}_{1,1}=b y_{1,1}+a y_{1,2}+v_{1} \\
& \ddot{y}_{1,2}=a y_{1,1}+b y_{1,2}+a y_{1,3}+v_{2} \\
& \ddot{y}_{1,3}=a y_{1,2}+b y_{1,3}+a y_{1,4}+v_{3} \\
& \ldots \\
& \ldots
\end{aligned}
$$

$\ddot{y}_{1, N-1}=a y_{1, N-2}+b y_{1, N-1}+a y_{1, N}+v_{N-1}$

$\ddot{y}_{1, N}=a y_{1, N-1}+b y_{1, N}+v_{N}$

For the dynamics given in Eq. (36), the feedback control law that assures tracking of the reference setpoint $y^{d}=$ $\left[y_{1,1}^{d}, y_{1,2}^{d}, y_{1,3}^{d}, \ldots, y_{1, N-1}^{d}, y_{1, N}^{d}\right]^{T}$ is

$$
\begin{aligned}
v_{1}= & \ddot{y}_{1,1}^{d}-b y_{1,1}-a y_{1,2}-K_{d_{1,1}}\left(\dot{y}_{1,1}^{d}-\dot{y}_{1,1}^{d}\right) \\
& -K_{p_{1,1}}\left(y_{1,1}^{d}-y_{1,1}^{d}\right) \\
v_{2}= & \ddot{y}_{1,2}^{d}-a y_{1,1}-b y_{1,2}-a y_{1,3}-K_{d_{1,2}}\left(\dot{y}_{1,2}^{d}-\dot{y}_{1,2}^{d}\right) \\
& -K_{p_{1,2}}\left(y_{1,2}^{d}-y_{1,2}^{d}\right) \\
v_{3}= & \ddot{y}_{1,3}^{d}-a y_{1,2}-b y_{1,3}-a y_{1,4}-K_{d_{1,3}}\left(\dot{y}_{1,3}^{d}-\dot{y}_{1,3}^{d}\right) \\
- & K_{p_{1,3}}\left(y_{1,3}^{d}-y_{1,3}^{d}\right) \\
\ldots & \quad-\quad-K_{d_{1, N-1}}\left(\dot{y}_{1, N-1}^{d}-\dot{y}_{1, N-1}^{d}\right) \\
\ldots & \quad-K_{p_{1, N-1}}\left(y_{1, N-1}^{d}-y_{1, N-1}^{d}\right) \\
v_{N-1}= & \ddot{y}_{1, N-1}^{d}-a y_{1, N-2}-b y_{1, N-1}-a y_{1, N} \\
\quad & \ddot{y}_{1, N}^{d}-a y_{1, N-1}-b y_{1, N}-K_{d_{1, N}}\left(\dot{y}_{1, N}^{d}-\dot{y}_{1, N}^{d}\right) \\
& -K_{p_{1, N}\left(y_{1, N}^{d}-y_{1, N}^{d}\right)}
\end{aligned}
$$

Next, using Eq. (37) one can compute the control action that is applied to the wave-type dynamics

$$
\begin{aligned}
v_{1} & =\frac{K}{\Delta x^{2}} \phi_{0}+f\left(y_{1,1}\right)+u\left(y_{1,1}\right) \\
& \Rightarrow u\left(y_{1,1}\right)=v_{1}-\frac{K}{\Delta x^{2}} \phi_{0}-f\left(y_{1,1}\right) \\
v_{2} & =f\left(y_{1,2}\right)+u\left(y_{1,2}\right) \Rightarrow, u\left(y_{1,2}\right)=v_{2}-f\left(y_{1,2}\right) \\
v_{3} & =f\left(y_{1,3}\right)+u\left(y_{1,3}\right) \Rightarrow u\left(y_{1,3}\right)=v_{3}-f\left(y_{1,3}\right) \\
\ldots &
\end{aligned}
$$




$$
\begin{aligned}
v_{N-1} & =f\left(y_{1, N-1}\right)+u\left(y_{1, N-1}\right) \\
& \Rightarrow u\left(y_{1, N-1}\right)=v_{N-1}-f\left(y_{1, N-1}\right) \\
v_{N}= & \frac{K}{\Delta x^{2}} \phi_{N+1}+f\left(y_{N}\right)+u\left(y_{1, N}\right) \\
\Rightarrow & u\left(y_{1, N}\right)=v_{N}-\frac{K}{\Delta x^{2}} \phi_{N+1}-f\left(y_{1, N}\right)
\end{aligned}
$$

\subsection{Solution of the Estimation Problem}

By selecting measurements from a subset of points $x_{j} j \in[1,2, \ldots, m]$, the associated observation (measurement) equation remains as in Eq. (32), i.e.

$$
\left(\begin{array}{c}
z_{1} \\
z_{2} \\
\cdots \\
z_{m}
\end{array}\right)=\left(\begin{array}{cccccc}
1 & 0 & 0 & \cdots & 0 & 0 \\
0 & 0 & 0 & \cdots & 0 & 0 \\
\cdots & \cdots & \cdots & \cdots \\
0 & 0 & 0 & \cdots & 1 & 0 \\
0 & 0 & 0 & \cdots & 0 & 0
\end{array}\right)\left(\begin{array}{c}
y_{1,1} \\
y_{2,1} \\
y_{1,2} \\
y_{2,2} \\
\cdots \\
y_{1, N} \\
y_{2, N}
\end{array}\right)
$$

For the description of the system in the form of Eq. (33) one can perform estimation using the Derivative-free nonlinear Kalman Filter recursion. In the filter's algorithm, the previously defined matrices $A, B$ and $C$ are substituted by their discrete-time equivalents $A_{d}, B_{d}$ and $C_{d}$. The discrete-time Kalman filter can be decomposed into two parts: (i) time update (prediction stage), and (ii) measurement update (correction stage).

\section{measurement update}

$$
\begin{aligned}
& K(k)=P^{-}(k) C_{d}^{T}\left[C \cdot P^{-}(k) C_{d}^{T}+R\right]^{-1} \\
& \hat{y}(k)=\hat{y}^{-}(k)+K(k)\left[z(k)-C_{d} \hat{y}^{-}(k)\right] \\
& P(k)=P^{-}(k)-K(k) C_{d} P^{-}(k)
\end{aligned}
$$

\section{time update}

$$
\begin{aligned}
& P^{-}(k+1)=A_{d}(k) P(k) A_{d}^{T}(k)+Q(k) \\
& \hat{y}^{-}(k+1)=A_{d}(k) \hat{y}(k)+B_{d}(k) u(k)
\end{aligned}
$$

Therefore, by taking measurements of $\phi(x, t)$ at time instant $t$ at a small number of measuring points $j=1, \ldots, n_{1}$ it is possible to estimate the complete state vector, i.e. to get values of $\phi$ in a mesh of points that covers efficiently the variations of $\phi(x, t)$. By processing a sequence of output measurements of the system, one can obtain local estimates of the state vector $\hat{y}$. The measuring points (active sensors) can vary in time provided that the observability criterion for the state-space model of the PDE holds.

According to Eqs. (40) and (41) an estimate of the system's state vector $\hat{y}$ is obtained. Should one want to obtain an

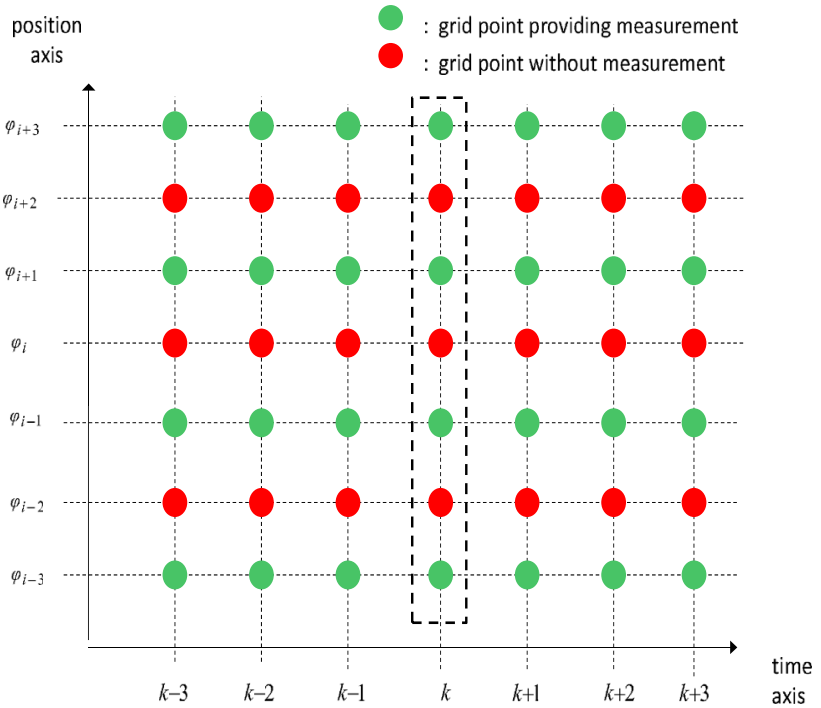

Fig. 3 Grid points for measuring $\phi(x, t)$

estimate of the state vector $x$ of the initial state-space description of the nonlinear wave-type dynamics he should apply the inverse differential flatness transformation connecting $x_{i}$ 's to $y_{i}$ 's. In the examined model of the wave PDE it holds that

$$
\begin{array}{lll}
\hat{x}_{1}=\hat{y}_{1,1} & \hat{x}_{2}=\hat{y}_{1,2} & \hat{x}_{3}=\hat{y}_{1,3} \\
\cdots & \cdots & \cdots \\
\hat{x}_{N-2}=\hat{y}_{1, N-2} & \hat{x}_{N-1}=\hat{y}_{1, N-1} & \hat{x}_{N}=\hat{y}_{1, N}
\end{array}
$$

Remark The proposed derivative-free nonlinear Kalman Filter is of improved precision because unlike other nonlinear filtering schemes, e.g. unlike the Extended Kalman Filter it does not introduce cumulative numerical errors due to approximative linearization of the system's dynamics. Besides it is computationally more efficient (faster) because it does not require to calculate Jacobian matrices and partial derivatives (Fig. 3).

If state estimation-based control is applied to the statespace model of the nonlinear wave-PDE then the associated control inputs are

$$
\begin{aligned}
& v_{1}=\frac{K}{\Delta x^{2}} \phi_{0}+f\left(\hat{y}_{1,1}\right)+u\left(\hat{y}_{1,1}\right) \\
& \quad \Rightarrow u\left(\hat{y}_{1,1}\right)=v_{1}-\frac{K}{\Delta x^{2}} \phi_{0}-f\left(\hat{y}_{1,1}\right) \\
& v_{2}=f\left(\hat{y}_{1,2}\right)+u\left(\hat{y}_{1,2}\right) \Rightarrow u\left(\hat{y}_{1,2}\right)=v_{2}-f\left(\hat{y}_{1,2}\right) \\
& v_{3}=f\left(\hat{y}_{1,3}\right)+u\left(\hat{y}_{1,3}\right) \Rightarrow u\left(\hat{y}_{1,3}\right)=v_{3}-f\left(\hat{y}_{1,3}\right) \\
& \ldots \\
& \ldots \quad \\
& v_{N-1}=f\left(\hat{y}_{1, N-1}\right)+u\left(\hat{y}_{1, N-1}\right) \\
& \quad \Rightarrow u\left(\hat{y}_{1, N-1}\right)=v_{N-1}-f\left(\hat{y}_{1, N-1}\right)
\end{aligned}
$$



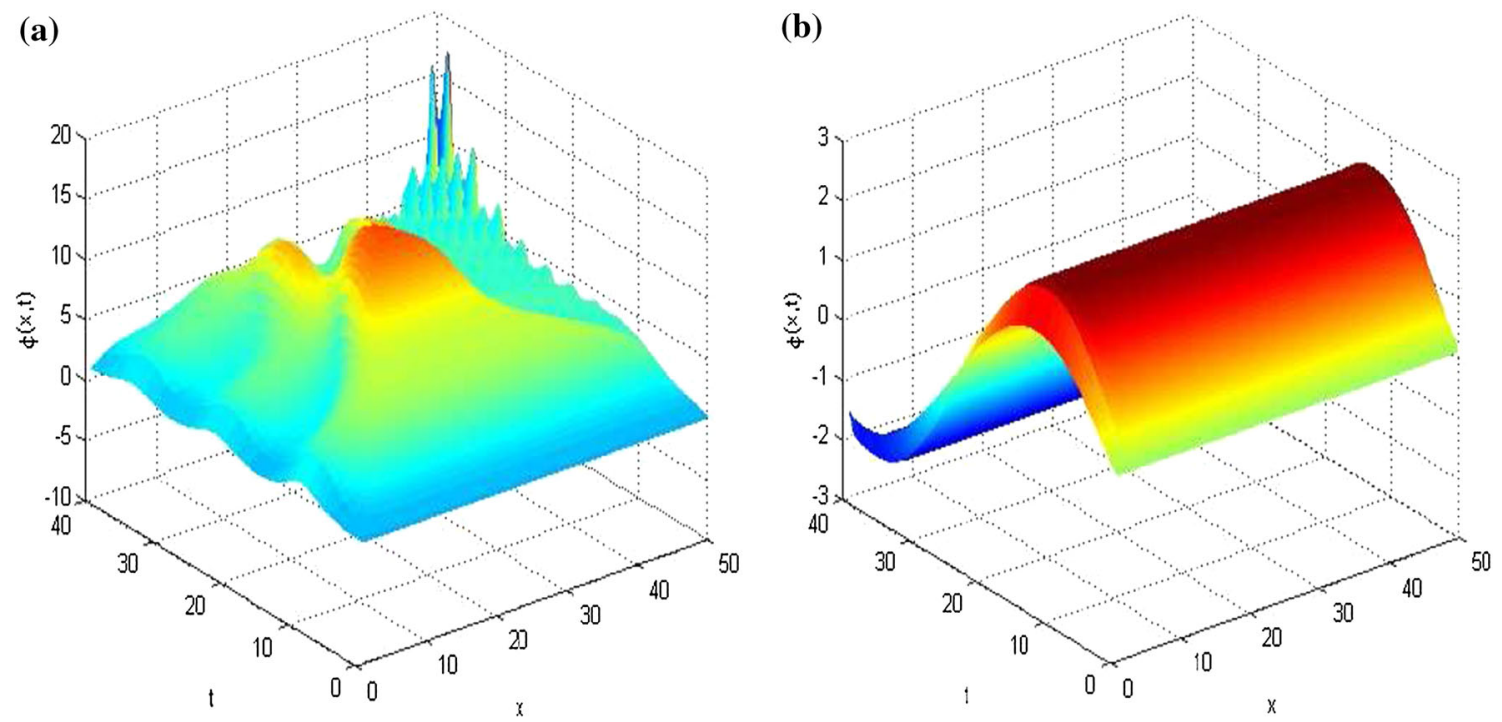

Fig. 4 a Nonlinear wave dynamics $\phi(x, t)$ without control. b Nonlinear wave dynamics $\phi(x, t)$ made to track a sinusoidal setpoint after applying control
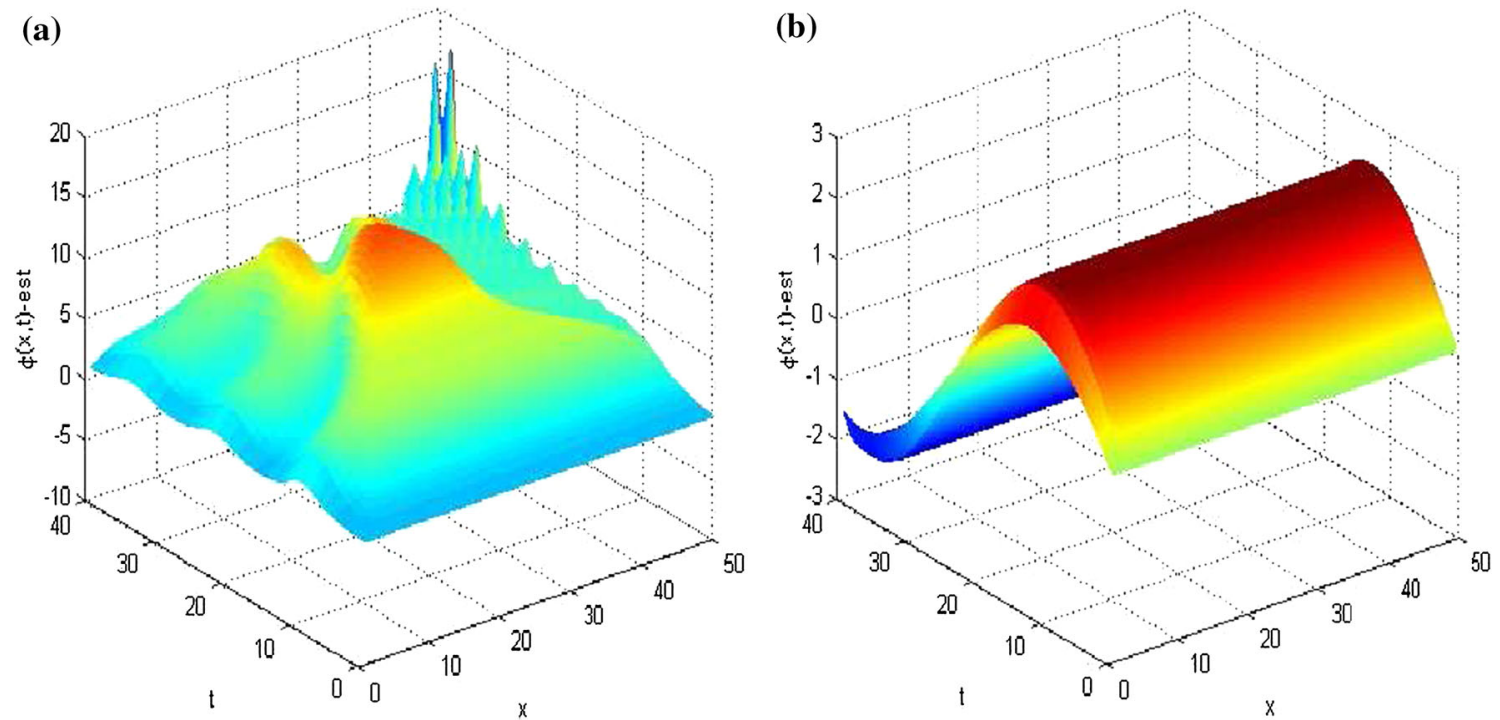

Fig. 5 State estimation-based control with the use of the derivative-free nonlinear Kalman Filter: a nonlinear wave dynamics $\phi(x, t)$ without control, b nonlinear wave dynamics $\phi(x, t)$ made to track a sinusoidal setpoint after applying control

$$
\begin{aligned}
v_{N} & =\frac{K}{\Delta x^{2}} \phi_{N+1}+f\left(\hat{y}_{N}\right)+u\left(\hat{y}_{1, N}\right) \\
& \Rightarrow u\left(\hat{y}_{1, N}\right)=v_{N}-\frac{K}{\Delta x^{2}} \phi_{N+1}-f\left(\hat{y}_{1, N}\right)
\end{aligned}
$$

\section{Simulation Tests}

The performance of the proposed control scheme has been tested in the model of the sine-Gordon nonlinear wave-type partial differential equation. A discretization grid of the PDE consisting of $N=50$ points was considered. At each grid point the local control input $u\left(x_{i}, t\right)$ was exerted to the system. The extended state-space description of the wavetype PDE comprised a state vector of dimension $y \in R^{100 \times 1}$. The number of measurement points was $m \leq N$ were $m$ was selected such that the observability of the linearized statespace model of the PDE is preserved. The obtained results are presented in Figs. 4, 5, 6 and 7. In Figs. 4 and 6 results are presented about control of the PDE dynamics using feedback of the entire state vector, while in Figs. 5 and 7 results about the state estimation-based control of the PDE are given. It can be observed that through the application of suitable control inputs the wave function $\phi(x, t)$ and its derivative $\dot{\phi}(x, t)$ can 

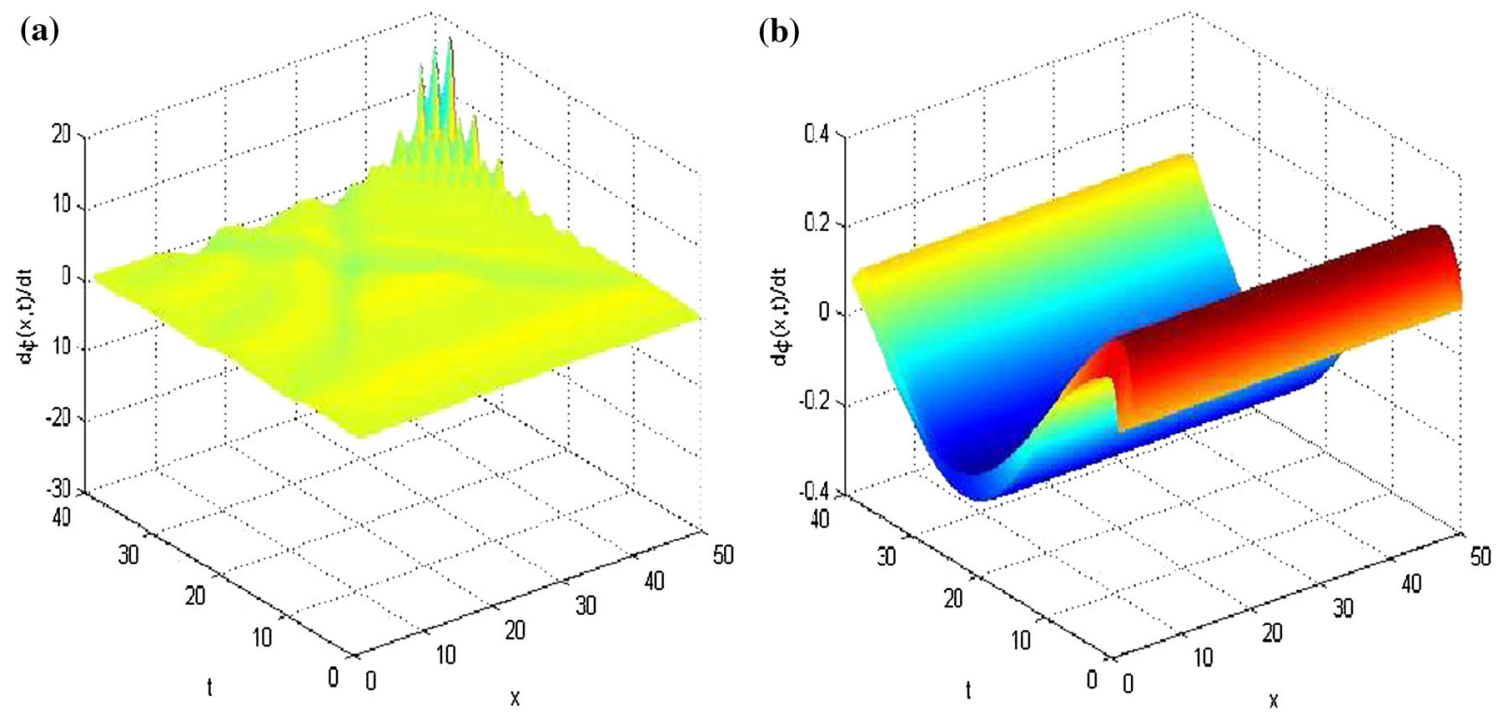

Fig. 6 a First time derivative of the nonlinear wave dynamics $\dot{\phi}(x, t)$ without control. b First time derivative of the nonlinear wave dynamics $\dot{\phi}(x, t)$ made to track a sinusoidal setpoint after applying control
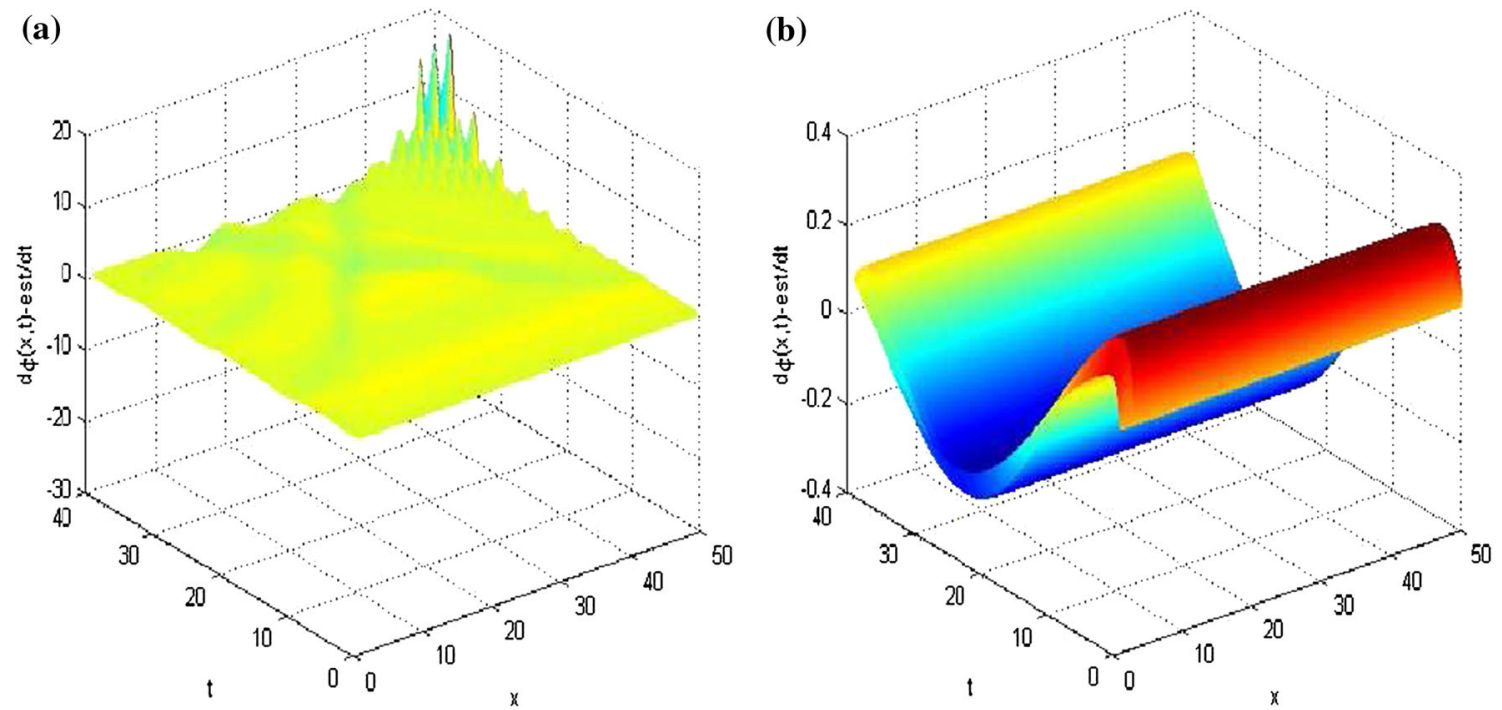

Fig. 7 State estimation-based control with the use of the derivative-free nonlinear Kalman Filter: a first time derivative of the nonlinear wave dynamics $\dot{\phi}(x, t)$ without control, b first time derivative of the nonlinear wave dynamics $\dot{\phi}(x, t)$ made to track a sinusoidal setpoint after applying control

be made to track the desirable sinusoidal setpoints. Moreover, in Figs. 8, 9, 10, 11 and 12 diagrams are provided about the tracking accuracy of the proposed control method at specific points of the discretization grid. It can be noticed again that the state estimation-based control method resulted in fast and accurate tracking of the reference setpoints.

\section{Conclusions}

A new nonlinear control and filtering method has been proposed for wave-type nonlinear partial differential equations.
After applying numerical methods for the discretization of the wave equation a state-space description of the associated PDE was obtained. It has been shown that this state space model of the wave-type nonlinear PDE is a differentially flat one. By proving that differential flatness properties hold and by applying a state variables transformation (diffeomorphism) it has been shown that the nonlinear wave-type PDE can be written as a state space-model of the linear canonical (Brunovsky) form. Thus, the model of the nonlinear wave-type PDE is represented by an equivalent set of linear ordinary differential equations (ODE). For the latter description the solution of the pointwise control problem becomes 

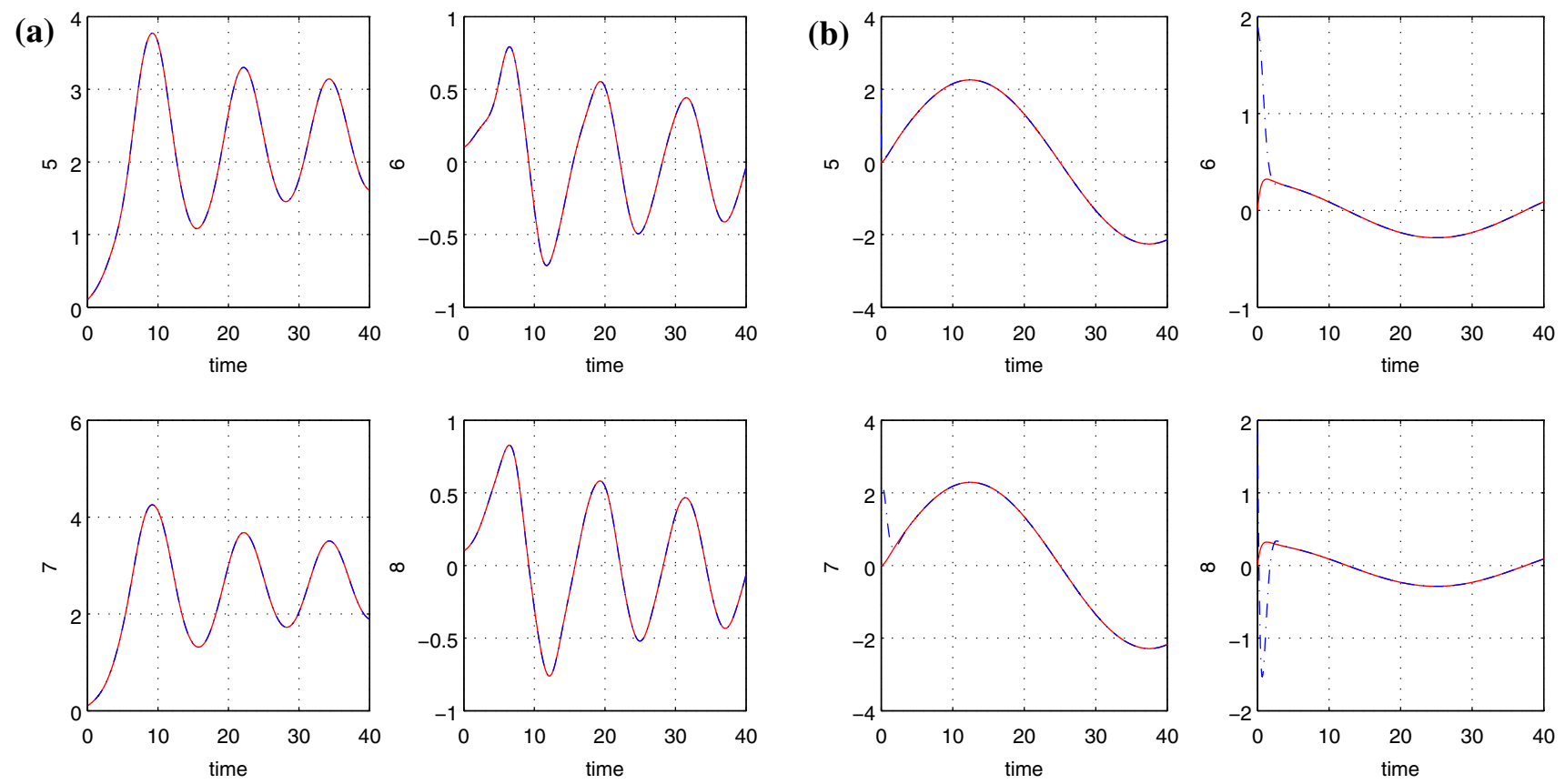

Fig. 8 Control and estimation of wave dynamics at grid points 3 (first row of diagrams) to 4 (second row of diagrams) a function $\phi(x, t)$ and its derivative when no control input is applied, $\mathbf{b}$ function $\phi(x, t)$ and its derivative under state feedback control
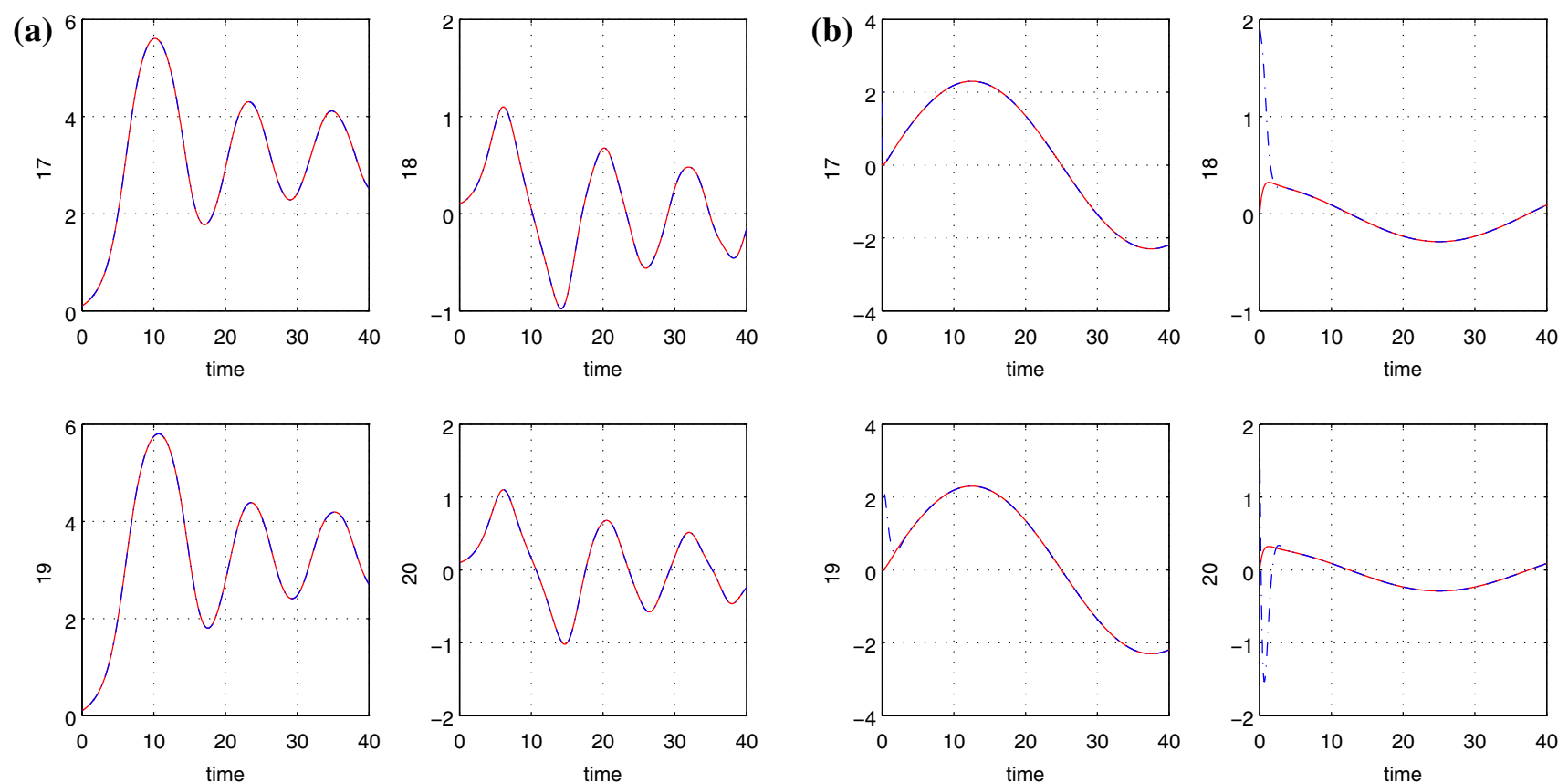

Fig. 9 Control and estimation of wave dynamics at grid points 9 (first row of diagrams) to 10 (second row of diagrams) a function $\phi(x, t)$ and its derivative when no control input is applied, $\mathbf{b}$ function $\phi(x, t)$ and its derivative under state feedback control

easy by applying a pole placement technique to each local ODE.

Another objective of the article has been to implement state-feedback control of the nonlinear wave dynamics using measurements from a small number of sensors. This has become possible through the application of a new nonlinear filtering method under the name Derivative-free nonlinear Kalman Filter. The filter comprises the standard Kalman Filter recursion applied to the linear equivalent state-space model of the wave PDE and of an inverse transformation 

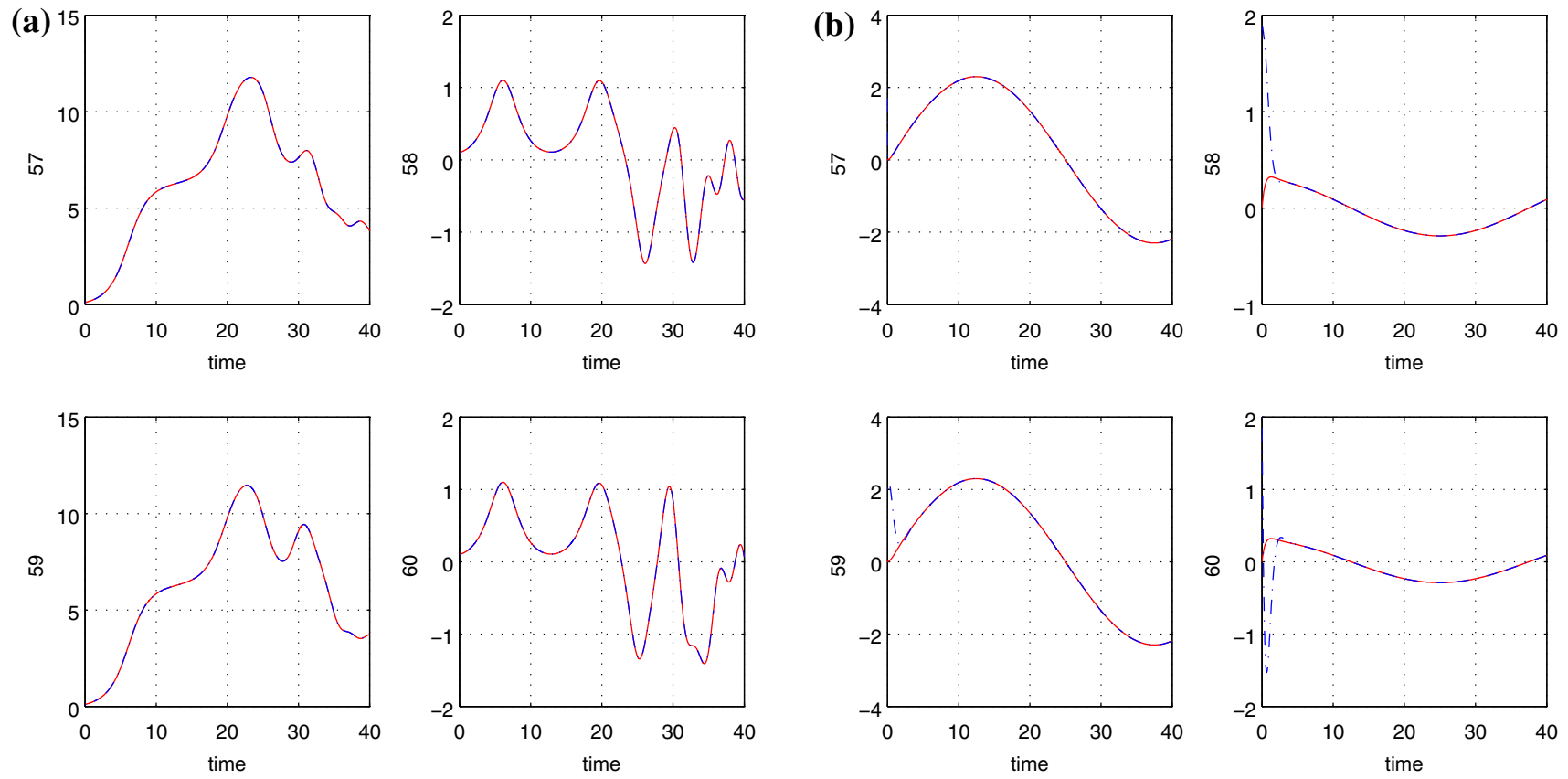

Fig. 10 Control and estimation of wave dynamics at grid points 29 (first row of diagrams) to 30 (second row of diagrams) a function $\phi(x, t)$ and its derivative when no control input is applied, $\mathbf{b}$ function $\phi(x, t)$ and its derivative under state feedback control
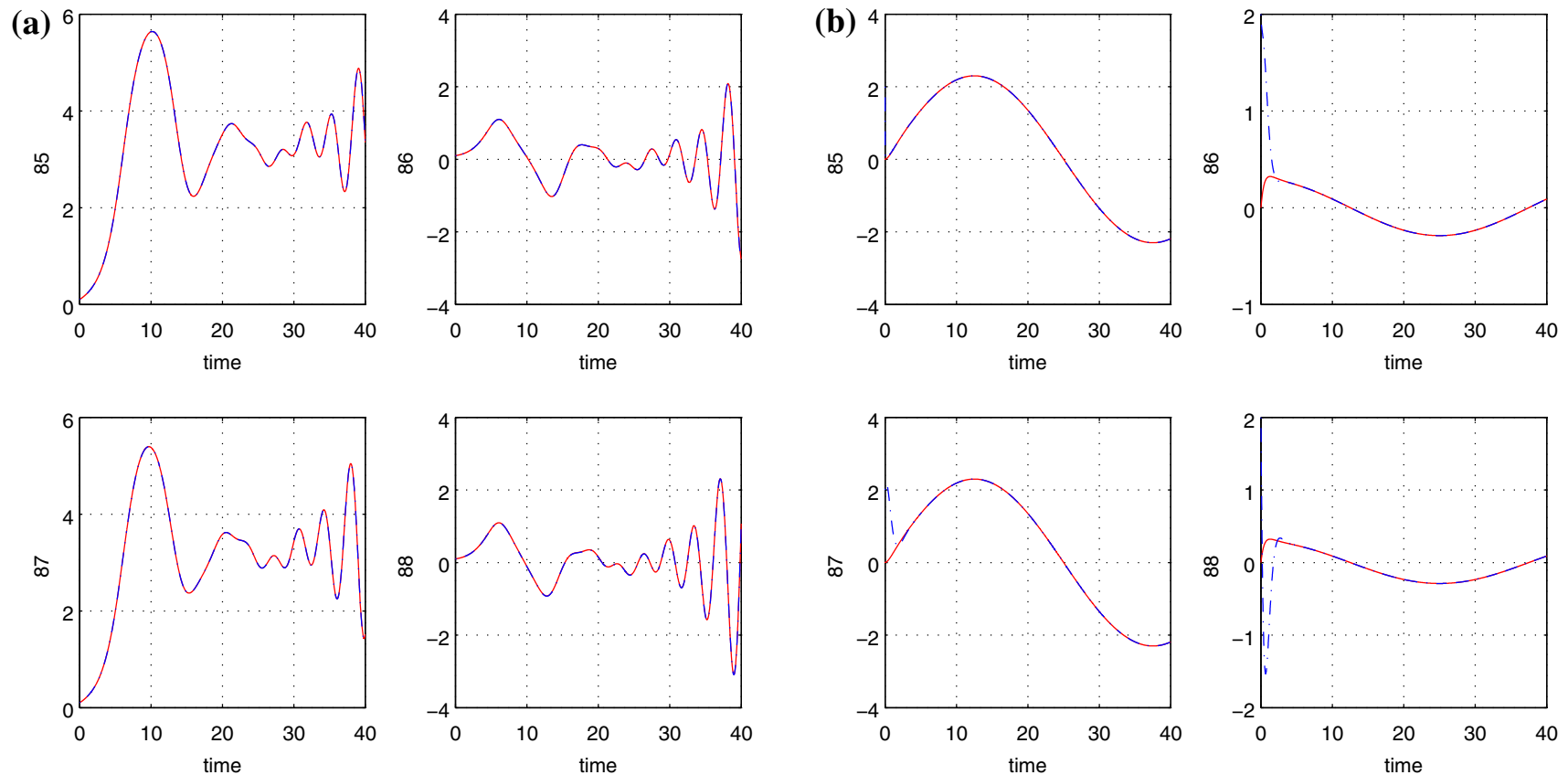

Fig. 11 Control and estimation of wave dynamics at grid points 43 (first row of diagrams) to 44 (second row of diagrams) a function $\phi(x, t)$ and its derivative when no control input is applied, $\mathbf{b}$ function $\phi(x, t)$ and its derivative under state feedback control

which is based on differential flatness properties which provides estimates for the state variables of the initial nonlinear description of the system. The efficiency of the proposed control and filtering methods for nonlinear wave-type PDEs has been tested through simulation experiments. It is also noted that the method can be also applied to other distributed parameter systems of the parabolic, elliptic or hyperbolic type. 

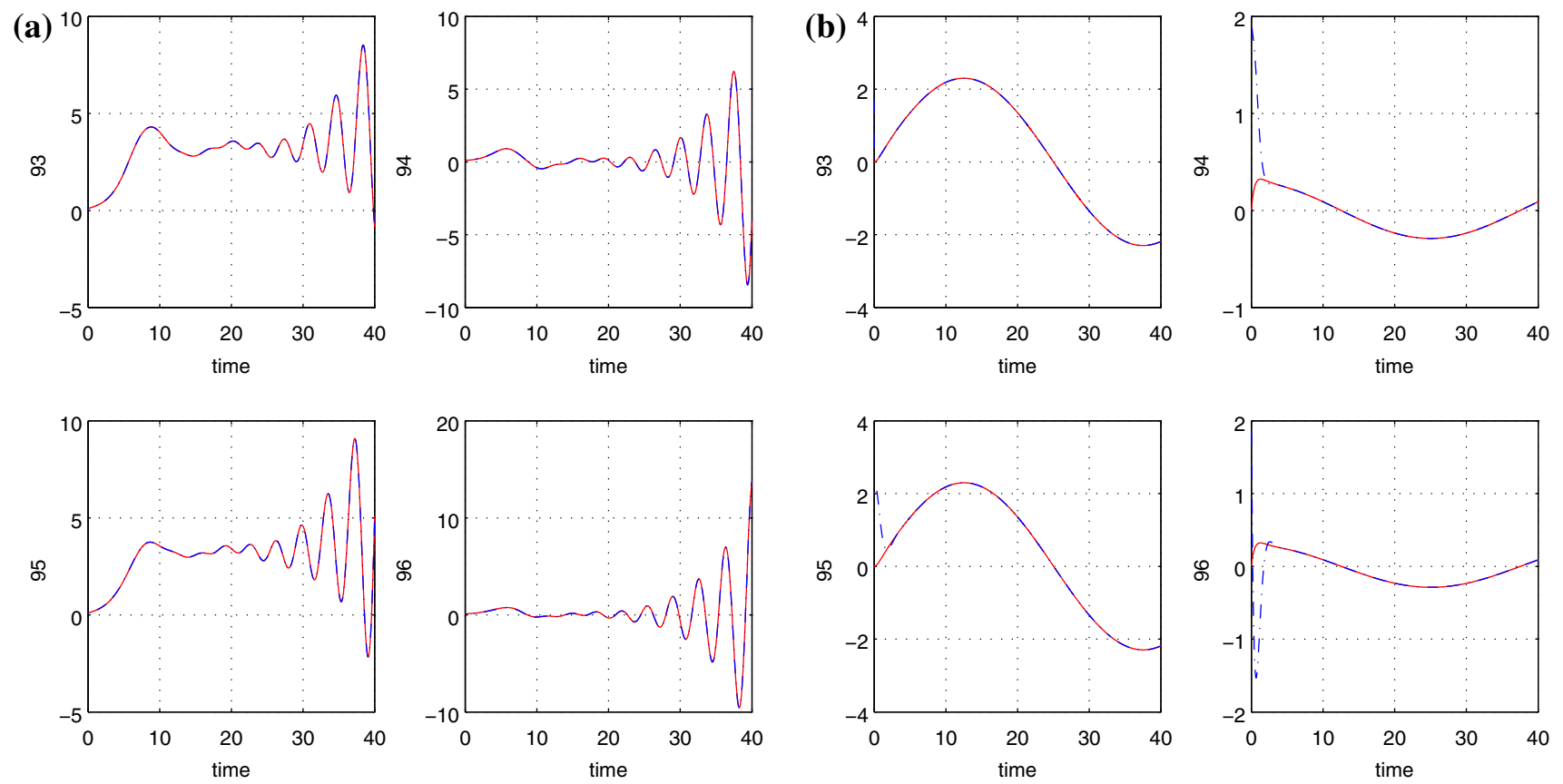

Fig. 12 Control and estimation of wave dynamics at grid points 47 (first row of diagrams) to 48 (second row of diagrams) a function $\phi(x, t)$ and its derivative when no control input is applied, $\mathbf{b}$ function $\phi(x, t)$ and its derivative under state feedback control

\section{References}

1. Rigatos, G.: Modelling and Control for Intelligent Industrial Systems: Adaptive Algorithms in Robotics and Industrial Engineering. Springer, Heidelberg (2011)

2. Rigatos, G.: Advanced Models of Neural Networks: Nonlinear Dynamics and Stochasticity of Biological Neurons. Springer, Heidelberg (2013)

3. Rigatos, G.: Nonlinear Control and Filtering Approaches Using Differential Flatness Theory: Applications to Electromechanical Systems. Springer, Cham (2015)

4. Gugat, M., Keiner, A., Leugering, G.: Optimal distributed control of the wave equation subject to state constraints. ZAMM Z. Angev. Math. Mech. 89(6), 420-444 (2009)

5. Maidi, A., Corriou, J.P.: Distributed control of nonlinear diffusion systems by input-output linearization. Int. J. Robust Nonlinear Control 26, 389-405 (2014)

6. Lions, J.L.: Pointwise control for distributed systems. In: Banks, H.T. (ed.) Control and Estimation in Distributed Parameter Systems. SIAM Frontiers in Applied Mathematics, Philadelphia (1992)

7. Dragonescu, A., Soane, A.M.: Multigrid solution of a distibuted optimal control problem constrained by the Stokes equations. Appl. Math. Comput. Elsevier 219, 5622-5634 (2013)

8. Winkler, F.J., Krause, I., Lohmann, B.: Flatness-based control of a continuous furnace. In: 18th International Conference on Control Applications, Part of 2009 IEEE Multi-Conference on Systems and Control, Saint Petersburg (2009)

9. Winkler, F.J., Lohmann, B.: Design of a decoupling controller structure for first order hyperbolic PDEs with distributed control action. In: 2010 American Control Conference, Baltimore (2010)

10. Saadatpour, A., Levi, M.: Travelling waves in chains of pendula. Phys. D 244, 68-73 (2013)
11. Guo, L., Billings, S.A.: State-space reconstruction and spatiotemporal prediction of lattice dynamical systems. IEEE Trans. Autom. Control 52(4), 622-632 (2007)

12. Maidi, A., Corriou, J.P.: Boundary control of nonlinear distributed parameter systemsby input-output linearization. In: 18th IFAC World Congress, Milan (2011)

13. Zuazua, E.: Propagation, observation and control of waves approximated by finite difference methods. SIAM Rev. 47(2), 197-243 (2005)

14. Kröner, A.: Adaptive finite element methods for optimal control second order hyberbolic equations. Comput. Methods Appl. Math. 11(2), 214-240 (2011)

15. Gerdes, M., Greif, G., Peich, H.J.: Numerical optimal control of the wave equation: optimal boundary control of a string to rest in finite time. In: Proceedings of the 5th Mathmod Conference, Vienna (2006)

16. Krstic, M.: Adaptive control of an anti-stable wave PDE. Dyn. Contin. Discret. Impuls. Syst. Ser. A 17, 853-882 (2010)

17. Krstic, M., Guo, B.Z., Balogh, A., Smyshlyaev, A.: Outputfeedback stabilization o an unstable wave equation. Automatica 44, 63-74 (2008)

18. Pinsky, M.: Partial Differential Equations and Boundary Value Problems. Prentice-Hall, Upper Saddle River (1991)

19. Mounier, H., Rudolph, J.: Trajectory tracking for $\pi$-flat nonlinear dealy systems with a motor example. In: Isidori, A., LamnabhiLagarrigue, F., Respondek, W. (eds). Nonlinear control in the year 2000, vol.1, Lecture Notes in Control and Inform. Sci., vol. 258, pp. 339-352, Springer (2001)

20. Rudolph, J.: Flatness Based Control of Distributed Parameter Systems, Steuerungs- und Regelungstechnik. Shaker Verlag, Aachen (2003)

21. Lévine, J.: On necessary and sufficient conditions for differential flatness. Appl. Algebra Eng. Commun. Comput. 22(1), 47-90 (2011) 
22. Fliess, M., Mounier, H.: Tracking control and $\pi$-freeness of infinite dimensional linear systems. In: Picci, G., Gilliam, D.S. (eds.) Dynamical Systems, Control, Coding and Computer Vision, vol. 258, pp. 41-68. Birkhaüser, Boston (1999)

23. Bououden, S., Boutat, D., Zheng, G., Barbot, J.P., Kratz, F.: A triangular canonical form for a class of 0-flat nonlinear systems. Int. J. Control Taylor Fr. 84(2), 261-269 (2011)

24. Rigatos, G.G.: A derivative-free Kalman Filtering approach to state estimation-based control of nonlinear dynamical systems. IEEE Trans. Ind. Electron. 59(10), 3987-3997 (2012)

25. Marino, R., Tomei, P.: Global asymptotic observers for nonlinear systems via filtered transformations. IEEE Trans. Autom. Control 37(8), 1239-1245 (1992)

26. Woittennek, F., Rudolph, J.: Controller canonical forms and flatness-based state feedback for 1D hyperbolic systems. In: 7th Vienna International Conference on Mathematical Modelling, MATHMOD (2012)

27. Bertoglio, C., Chapelle, D., Fernandez, M.A., Gerbeau, J.F., Moireau, P.: State observers of a vascular fluid-structure interaction model through measurements in the solid. In: INRIA research report no 8177 (2012)

28. Salberg, S.A., Maybeck, P.S., Oxley, M.E.: Infinite-dimensional sampled-data Kalman Filtering and stochastic heat equation. In: 49th IEEE Conference on Decision and Control, Atlanta, Georgia (2010)

29. Yu, D., Chakravotry, S.: A randomly perturbed iterative proper orthogonal decomposition technique for filtering distributed parameter systems. American Control Conference, Montreal (2012)
30. Haine, G.: Observateurs en dimension infinie. Application à 1 étude de quelques problèmes inverses. Thèse de doctorat. Institut Elie Cartan Nancy (2012)

31. Hidayat, Z., Babuska, R., de Schutter, B., Nunez, A.: Decentralized Kalman Filter comparison for distributed parameter systems: a case study for a 1D heat conduction process. In: Proceedings of the 16th IEEE International Conference on Emerging Technologies and Factory Automatio, ETFA 2011, Toulouse (2011)

32. Demetriou, M.A.: Design of consensus and adaptive consensus filters for distributed parameter systems. Automatica 46, 300-311 (2010)

33. Guo, B.Z., Xu, C.Z., Hammouri, H.: Output feedback stabilization of a one-dimensional wave equation with an arbitrary time-delay inboundary observation. ESAIM Control Optim. Calc. Var. 18, 2225 (2012)

34. Chauvin, J.: Observer design for a class of wave equations driven by an unknown periodic input. In: 18th World Congress, Milano (2011)

35. Rigatos, G.G., Tzafestas, S.G.: Extended Kalman filtering for fuzzy modelling and multi-sensor fusion. Math. Comput. Model. Dyn. Syst. 13, 251-266 (2007)

36. Basseville, M., Nikiforov, I.: Detection of Abrupt Changes: Theory and Applications. Prentice-Hall, Englewood Cliffs (1993)

37. Rigatos, G., Zhang, Q.: Fuzzy model validation using the local statistical approach. Fuzzy Sets Syst. 60(7), 882-904 (2009) 